\title{
NOETHERIAN PROPERTIES OF SKEW POLYNOMIAL RINGS WITH BINOMIAL RELATIONS
}

\author{
TATIANA GATEVA-IVANOVA
}

\begin{abstract}
In this work we study standard finitely presented associative algebras over a fixed field $K$. A restricted class of skew polynomial rings with quadratic relations considered in an earlier work of $\mathbf{M}$. Artin and W. Schelter will be studied. We call them binomial skew polynomial algebras. We establish necessary and sufficient conditions for such an algebra to be a Noetherian domain.
\end{abstract}

\section{INTRODUCTION}

In this paper we work with graded associative algebras over a fixed field $K$. Given a nonempty set $X=\left\{x_{1}, \ldots, x_{n}\right\},\langle X\rangle$ will denote the free monoid with unit generated by $X, K\langle X\rangle$ will denote the free associative algebra (with 1) generated by $X$. We fix the degree-lexicographic order $\langle *$ on $\langle X\rangle$ (we set $\left.x_{1}<* x_{2}<* \cdots<* x_{n}\right)$.

(1.1) An ordered monomial in $\left\{x_{1}, \ldots, x_{n}\right\}$ is a monomial of the type $x_{1}^{t_{1}} \ldots$ $x_{n}^{t_{n}}, t_{i} \geq 0$. By $N$ we shall denote the set of all ordered monomials. Given a polynomial $f$ in $K\langle X\rangle, H M(f)$ will denote its highest monomial. For any subset $F$ of $K\langle X\rangle,(F)$ will denote the two-sided ideal generated by $F$.

(1.2) Let $F_{0}$ be a set of polynomials in $K\langle X\rangle$ of the type

$$
F_{0}=\left\{x_{j} x_{i}-f_{j i} \mid 1 \leq i<j \leq n\right\},
$$

where for $1 \leq i<j \leq n, f_{j i}$ is a linear combination of ordered monomials of degree 2, and $H M\left(f_{j i}\right)<* x_{j} x_{i}$. We shall recall the following

(1.3) Definition. A monomial $u$ is normal (modulo $F_{0}$ ) if it does not contain as a segment any of the monomials $x_{j} x_{i}, 1 \leq i<j \leq n$.

It is clear that a monomial is normal $\left(\bmod F_{0}\right)$ if and only if it is an ordered monomial.

We shall recall now some facts extracted from Bergman's Diamond Lemma [Berg] in the particular case, when $\langle X\rangle$ is ordered by the degree-lexicographic ordering, and the set $F_{0}$ is as in (1.2).

Received by the editors March 1, 1991 and, in revised form, July 5, 1992.

1991 Mathematics Subject Classification. Primary 16S15, 16P40; Secondary 16S36.

Partially supported by the Ministry of Science and Education (Bulgaria) and the National Science Foundation (U.S.A.) under the joint programme "Algebra and Algebraic Geometry". 
Consider the $K$-linear operators (we call them reductions)

$$
r_{u, j, i, v}, \quad \text { for } u, v \in\langle X\rangle, \quad 1 \leq i<j \leq n,
$$

defined on the underlying vector space of $K\langle X\rangle$ by the formulas

$$
\begin{aligned}
r_{u, j, i, v}\left(u x_{j} x_{i} v\right) & =u f_{j i} v, \\
r_{u, j, i, v}(w) & =w, \quad \text { for all } w \neq u x_{j} x_{i} v .
\end{aligned}
$$

It is well known, cf. [Berg], that for any polynomial $f$ in $K\langle X\rangle$ there exists a finite sequence of reductions, $r_{1}, r_{2}, \ldots, r_{s}$, such that

$$
f \stackrel{r_{1}}{\rightarrow} f_{1} \stackrel{r_{2}}{\rightarrow} f_{2} \rightarrow \cdots \stackrel{r_{s}}{\rightarrow} f_{s},
$$

where $f-f_{s}$ is in the ideal $\left(F_{0}\right)$, and $f_{s}$ is a linear combination of ordered (or equivalently normal) monomials. In general $f_{s}$ is not uniquely determined. It follows from Bergman's Diamond Lemma that

(1.4) [Berg]. The following conditions are equivalent:

(a) There is an isomorphism of vector spaces $K\langle X\rangle \cong \operatorname{Span} N \oplus\left(F_{0}\right)$;

(b) For any triple $(k, j, i)$, where $n \geq k>j>i \geq 1$, the difference $f_{k j} x_{i}-x_{k} f_{j i}$ can be reduced to zero (by means of a finite sequence of reductions).

In particular, if this is the case for $F_{0}$, the set of ordered monomials $N$ projects to a basis (as a vector space) of the algebra $A=K\langle X\rangle /\left(F_{0}\right)$.

(1.5) It is clear that in this case for any polynomial $f$ in $K\langle X\rangle$, one has $f=$ $\operatorname{Nor}(f)+h$, where $\operatorname{Nor}(f) \in \operatorname{Span} N$, and $h \in\left(F_{0}\right)$ are uniquely determined. The element $\operatorname{Nor}(f)$ is called the normal form of $f$.

(1.6) Definition. The set $F_{0}$ is called a Groebner basis for the ideal $\left(F_{0}\right)$ if it satisfies the equivalent conditions (1.4(a), (b)).

For general references on Groebner bases see [Berg, Buch, Gol, G-I $\mathrm{I}_{1}$, Mor ${ }_{1}$, $\mathrm{Mor}_{2}$ and K-R-W].

(1.7) Definition [Art-S]. An algebra $A$ is a skew polynomial ring if it can be presented as $A=K\langle X\rangle /\left(F_{0}\right)$, where the set of relations $F_{0}$ is as in (1.2) and is a Groebner basis of the ideal $\left(F_{0}\right)$.

In this paper we shall work with a particular case of skew polynomial rings, namely with "binomial" skew polynomial rings.

(1.8) A skew polynomial ring $A=K\langle X\rangle /(F)$ is called binomial if the Groebner basis $F$ is of the form:

$$
\begin{aligned}
F=\left\{x_{j} x_{i}-a_{i j} x_{i^{\prime}} x_{j^{\prime}} \mid 1 \leq i<j \leq n\right\} \\
\text { where } 0 \neq a_{i j} \in K, 1 \leq i^{\prime} \leq j^{\prime} \leq n, x_{i^{\prime}} x_{j^{\prime}}<* x_{j} x_{i} .
\end{aligned}
$$

(1.10) We shall always assume that $i^{\prime}<j^{\prime}$, excluding the case $i^{\prime}=j^{\prime}$.

Note that in general the set $\left\{x_{i^{\prime}} x_{j^{\prime}} \mid 1 \leq i<j \leq n\right\}$ contains at most $\left(\begin{array}{l}n \\ 2\end{array}\right)$ elements; i.e., we do not assume that all the monomials $x_{i^{\prime}} x_{j^{\prime}}$, for $1 \leq i<j \leq$ $n$, are pairwise different. 
Examples.

$$
\begin{gathered}
A=K\left\langle x_{1}, x_{2}, x_{3}\right\rangle /\left(x_{3} x_{2}-x_{2} x_{3}, x_{3} x_{1}-x_{1} x_{2}, x_{2} x_{1}-x_{1} x_{2}\right), \\
A=K\left\langle x_{1}, x_{2}, x_{3}\right\rangle /\left(x_{3} x_{2}-x_{1} x_{3}, x_{3} x_{1}-x_{1} x_{3}, x_{2} x_{1}-x_{1} x_{2}\right), \\
A=K\left\langle x_{1}, x_{2}, x_{3}, x_{4}\right\rangle /\left(x_{4} x_{3}-x_{3} x_{4}, x_{4} x_{2}-x_{2} x_{4}, x_{4} x_{1}-x_{1} x_{3},\right. \\
\left.x_{3} x_{2}-x_{2} x_{3}, x_{3} x_{1}-x_{1} x_{2}, x_{2} x_{1}-x_{1} x_{4}\right) .
\end{gathered}
$$

We are interested in the question when a binomial skew polynomial algebra $A$ is Noetherian.

There are various results on the Noetherianness of algebras with quadratic relations [Ap, G-I 1 , G-I ${ }_{2}$, Mor $_{1}, \mathrm{~K}-\mathrm{R}-\mathrm{W}, \mathrm{Sm}-\mathrm{St}$ ].

Before formulating the main results of this paper we need some more notation. From now on we shall always assume that the set $F$ is fixed. It is clear that the normal form of any monomial $w$ in $\langle X\rangle$ is of the type $c w_{0}$, where $c \in K$ and $w_{0} \in N$.

(1.14) Given two normal monomials $u$ and $v$, by $u \star v$ we shall denote the monomial which appears in the normal form of $u \cdot v$. (In other words we ignore the coefficient appearing in the normal form of $u \cdot v$.) Clearly, for any pair $(j, i), 1 \leq i<j \leq n$, one has

$$
x_{j} \star x_{i}=x_{i^{\prime}} \cdot x_{j^{\prime}} \text {, where } i^{\prime} \text { and } j^{\prime} \text { are as in (1.9). }
$$

The main results of this paper are contained in Theorems $\mathrm{A}, \mathrm{B}$, and $\mathrm{C}$.

(1.16) Theorem A. Let $A=K\left\langle x_{1}, \ldots, x_{n}\right\rangle /(F)$ be a binomial skew polynomial ring without zero divisors, with reduced Groebner basis

$$
F=\left\{x_{j} x_{i}-a_{i j} x_{i^{\prime}} x_{j^{\prime}} \mid 1 \leq i<j \leq n\right\},
$$

where for $1 \leq i<j \leq n$ one has $1 \leq i^{\prime}<j^{\prime} \leq n$, and $x_{i^{\prime}} x_{j^{\prime}}<* x_{j} x_{i}$. Suppose furthermore that

$$
\text { The set }\left\{x_{i^{\prime}} x_{j^{\prime}} \mid 1 \leq i<j \leq n\right\} \text { contains precisely }\left(\begin{array}{l}
n \\
2
\end{array}\right) \text { elements. }
$$

Then the algebra $A$ is cyclic, i.e., the following condition is satisfied:

(1.18) For any $j$ and $k, 1 \leq k<j \leq n$, there exists $a p>k$ and a cycle $\sigma=\left(k, k_{1}, k_{2}, \ldots, k_{s}\right)$ in the symmetric group $S_{j}$, where all $k_{i}<p, j$, such that

$$
\begin{gathered}
x_{j} \star x_{k}=x_{k_{1}} \cdot x_{p}, \\
x_{j} \star x_{k_{1}}=x_{k_{2}} \cdot x_{p}, \\
\vdots \\
x_{j} \star x_{k_{s}}=x_{k} \cdot x_{p} .
\end{gathered}
$$

(1.19) Theorem B. Suppose $A=K\left\langle x_{1}, \ldots, x_{n}\right\rangle /(F)$ is a binomial skew polynomial ring, with reduced Groebner basis

$$
F=\left\{x_{j} x_{i}-a_{i j} x_{i^{\prime}} x_{j^{\prime}} \mid 1 \leq i<j \leq n\right\} .
$$

Suppose, furthermore, that for some positive integer $P$, the following condition is satisfied: 
(1.20) For any pair of integers $q, k, 1 \leq k<q \leq n$, there exists $a j, k<$ $j \leq n$, such that

$$
\left(x_{j}\right)^{P} \star x_{k}=x_{k} \cdot\left(x_{q}\right)^{P} .
$$

Then $A$ is left Noetherian.

(1.21) Theorem C. Let $A=K\left\langle x_{1}, \ldots, x_{n}\right\rangle /(F)$ be a binomial skew polynomial ring without zero divisors, with reduced Groebner basis

$$
F=\left\{x_{j} x_{i}-a_{i j} x_{i^{\prime}} x_{j^{\prime}} \mid 1 \leq i<j \leq n\right\},
$$

where for $1 \leq i<j \leq n$ one has $1 \leq i^{\prime}<j^{\prime} \leq n$, and $x_{i^{\prime}} x_{j^{\prime}}<* x_{j} x_{i}$. Then the following three conditions are equivalent:

(i) The set $\left\{x_{i^{\prime}} x_{j^{\prime}} \mid 1 \leq i<j \leq n\right\}$ contains precisely $\left(\begin{array}{l}n \\ 2\end{array}\right)$ elements.

(ii) $A$ is left Noetherian.

(iii) $A$ is right Noetherian.

(1.22) Remark. Further results on Noetherian binomial rings are obtained in [G- $\left.\mathrm{I}_{3}\right]$.

The work in this paper was done at MIT in the autumn and winter of 1990 under the auspices of the NSF-Bulgarian Academy of Sciences Exchange Program. I would like to express my gratitude to Michael Artin for his encouragement and support, and also for his attention to my work, George Bergman for some stimulating discussions and numerious helpful comments, and to Jim Carlson for his help on TeX matters.

\section{THE CYCLIC CONDITION}

In this section we shall prove Theorem A. All conventions and definitions made in the introduction shall be in force throughout the paper. We begin with some preliminary results, and we assume the hypotheses of the theorem.

(2.1) Remark. Since the Groebner basis $F$ consists of binomial relations only, it is clear that the normal form of any monomial $u$ is of the type $c \cdot v$ where $c$ is a nonzero coefficient in $K$ and $v$ is a normal monomial. It follows from the Diamond Lemma that both $c$ and $v$ are uniquely determined. We can ignore $c$ and write $u \rightarrow v$ to denote that $v$ is the result of "almost normalisation" of $u$. It is clear that in order to obtain $v$ it will be enough to use "almost reductions" instead of usual reductions, more precisely: For any $i, j, 1 \leq i<j \leq n$, we shall actually need a simpler "almost reduction" replacing $x_{j} \cdot x_{i}$ with $x_{i^{\prime}} \cdot x_{j^{\prime}}$ instead of replacing it by $a_{i j} \cdot x_{i^{\prime}} \cdot x_{j^{\prime}}$, which the usual reduction does.

For arbitrary monomials $v$ and $w$, we shall write $v \cdot\left[x_{j} \cdot x_{i}\right] \cdot w \rightarrow v \cdot\left(x_{i^{\prime}} \cdot\right.$ $\left.x_{j^{\prime}}\right) \cdot w$ to denote that we have replaced the monomial $x_{j} \cdot x_{i}$ by $x_{i^{\prime}} \cdot x_{j^{\prime}}$ (or equivalently by $\left.x_{j} \star x_{i}\right)$.

(2.2) Remark. Under the hypothesis of Theorem A it is clear that for any pair of integers $i, j, 1 \leq i<j \leq n$, there exist uniquely determined $q$ and $p$, $1 \leq p<q \leq n$, such that $x_{q} \star x_{p}=x_{i} \cdot x_{j}$.

(2.3) Lemma. Let $i$ and $j$ be integers, $1 \leq i<j \leq n$. Then for $i^{\prime}$ and $j^{\prime}$ as in (1.9), (1.10) one has $i^{\prime}<j$, and $i<j^{\prime}$.

Proof. The inequality $x_{i^{\prime}} x_{j^{\prime}}<* x_{j} x_{i}$ implies $i^{\prime} \leq j$. Thus $i^{\prime}<j$, since $A$ has no zero divisors. We shall prove now that $i<j^{\prime}$. Note first that $j^{\prime} \neq i$, 
since $A$ has no zero divisors. Assume that $j^{\prime}<i$. Consider the two possible ways of "almost normalisation" of the monomial $x_{j} \cdot x_{i} \cdot x_{j^{\prime}}$ :

$$
\left[x_{j} \cdot x_{i}\right] \cdot x_{j^{\prime}} \rightarrow\left(x_{i^{\prime}} \cdot x_{j^{\prime}}\right) \cdot x_{j^{\prime}}=u \in N,
$$

and

$$
x_{j} \cdot\left[x_{i} \cdot x_{j^{\prime}}\right] \rightarrow x_{j} \cdot\left(x_{r} \cdot x_{s}\right) \rightarrow \cdots \rightarrow u \in N
$$

where

$$
r<i, s .
$$

It follows from (1.10) that the final replacement in (2.5) should be of the type

$$
\left[x_{m} \cdot x_{k}\right] \cdot x_{j^{\prime}} \rightarrow\left(x_{i^{\prime}} \cdot x_{j^{\prime}}\right) \cdot x_{j^{\prime}},
$$

for some $m$ and $k, m>k$. It follows from here that

$$
x_{m} \star x_{k}=x_{i^{\prime}} \cdot x_{j^{\prime}}=x_{j} \star x_{i} .
$$

The inequality (2.6) and the fact that after any replacement the result is a monomial which is strictly less than the previous one, imply that the pairs $(m, k)$ and $(j, i)$ are different, thus (2.7) contradicts Remark (2.2).

Lemma (2.3) has been proved.

(2.8) Lemma (prohibiting an approach from below). Suppose $x_{j} \star x_{k}=x_{m} \cdot x_{p}$, where $j>k, p>m$. Suppose also that $x_{a} \star x_{b}=x_{k} \cdot x_{p}$, for some $a$ and $b$, $a>b$. Then $a \geq j$.

Proof. Assume the contrary, i.e., $a<j$. Consider the following replacements:

$$
x_{j} \cdot\left[x_{a} \cdot x_{b}\right] \rightarrow x_{j} \cdot\left(x_{k} \cdot x_{p}\right)=\left[x_{j} \cdot x_{k}\right] \cdot x_{p} \rightarrow\left(x_{m} \cdot x_{p}\right) \cdot x_{p}=u \in N
$$

and

$$
\left[x_{j} \cdot x_{a}\right] \cdot x_{b} \rightarrow f=\left(x_{v} \cdot x_{w}\right) \cdot x_{b} \rightarrow \cdots \rightarrow u
$$

where

$$
v<j
$$

It follows from (1.10) that as a final replacement in (2.10) one has

$$
g=\left[x_{s} \cdot x_{t}\right] \cdot x_{p} \rightarrow\left(x_{m} \cdot x_{p}\right) \cdot x_{p},
$$

where $s>t$, and

$$
x_{s} \star x_{t}=x_{m} \cdot x_{p}
$$

Since the monomial $g$ is obtained from $f$ as a result of a finite sequence of replacements (or $g=f$ ), one has

$$
f=x_{v} \cdot x_{w} \cdot x_{q} * \geq x_{s} \cdot x_{t} \cdot x_{p}=g,
$$

which implies $s \leq v$, and by (2.11) one has

$$
s<j .
$$

By the hypothesis of the lemma we have

$$
x_{j} \star x_{k}=x_{m} \cdot x_{p}
$$


which together with (2.12) implies

$$
x_{j} \star x_{k}=x_{s} \star x_{t}
$$

The pairs $(j, k)$ and $(s, t)$ are different by (2.13). Thus, by Remark (2.2) the equality (2.14) is impossible, a contradiction, due to the assumption that $a<j$. We have proved the lemma.

(2.15) Inductive Lemma. Under the hypothesis of Theorem A let $j_{0}$ be an integer, $1<j_{0}<n$, such that

(2.16) For any $j>j_{0}$ and $k, 1 \leq k<j$, there exists $a p>k$ and a cycle $\left(k, k_{1}, \ldots, k_{s}\right)$ such that

$$
\begin{aligned}
x_{j} \star x_{k} & =x_{k_{1}} \cdot x_{p}, \\
x_{j} \star x_{k_{1}} & =x_{k_{2}} \cdot x_{p}, \\
\vdots & \\
x_{j} \star x_{k_{s}} & =x_{k} \cdot x_{p} .
\end{aligned}
$$

Then the condition (2.16) holds for $j=j_{0}$.

Under the hypothesis of (2.15) we shall first prove some facts.

(2.17) Lemma. Let $k, j_{1}, j_{2}$, be integers such that $k \leq j_{0}<j_{1}, j_{2}, j_{1} \neq j_{2}$ and let

$$
x_{j_{1}} \star x_{k}=x_{k_{1}} \cdot x_{p_{1}} \text { and } x_{j_{2}} \star x_{k}=x_{k_{2}} \cdot x_{p_{2}},
$$

for some $k_{1}, k_{2}, p_{1}$, and $p_{2}$. Then $p_{1} \neq p_{2}$.

Proof. It follows from (2.16) that there exist $m_{1}$ and $m_{2}$ such that

$$
x_{j_{1}} \star x_{m_{1}}=x_{k} \cdot x_{p_{1}} \text { and } x_{j_{2}} \star x_{m_{2}}=x_{k} \cdot x_{p_{2}} .
$$

The pairs $\left(j_{1}, m_{1}\right),\left(j_{2}, m_{2}\right)$ are different since by hypothesis $j_{1} \neq j_{2}$. Thus, by $\operatorname{Remark}(2.2),. p_{1} \neq p_{2}$.

(2.18) Corollary. For any $j>j_{0}$ there exists an $a>j_{0}$, such that

$$
x_{a} \star x_{j_{0}}=x_{k} \cdot x_{j}
$$

for some $k, k<j$.

Proof. For $j_{0}<j \leq n$, let $p_{j}>j_{0}$ be the integer, determined by the equality

$$
x_{j} \star x_{j_{0}}=x_{k_{j}} \cdot x_{p_{j}} \in N, \text { for some } k_{j} \text {. }
$$

It follows from the previous lemma that all the elements $p_{n}, \ldots, p_{\left(j_{0}+1\right)}$ are pairwise different. Hence there is an equality of sets:

$$
\left\{p_{\left(j_{0}+1\right)}, \ldots, p_{n}\right\}=\left\{j_{0}+1, \ldots, n\right\},
$$

which proves the corollary.

(2.19) Lemma (taking the preimage). Let $k<j_{0}$, and let $x_{j_{0}} \star x_{k}=x_{k_{1}} \cdot x_{p}$, for some $p$ and $k_{1}, k_{1}<j_{0}$. Then there exists a uniquely determined $s<j_{0}$ such that

$$
x_{j_{0}} \star x_{s}=x_{k} \cdot x_{p}
$$


Proof. By Remark (2.2), there exist uniquely determined $j$ and $s, j>s$, such that $x_{j} \star x_{s}=x_{k} \cdot x_{p}$. It follows from Lemma (2.8) that $j \geq j_{0}$. Assume $j>j_{0}$. It follows then from (2.16) and (2.3) that

$$
x_{j} \star x_{k}=x_{k^{\prime}} \cdot x_{p}
$$

for some $k^{\prime}$,

$$
k^{\prime}<j, \quad k<p .
$$

By (2.18) there exists an $a>j_{0}$, such that

$$
x_{a} \star x_{j_{0}}=x_{q} \cdot x_{j}, \quad \text { for some } q .
$$

It follows from (2.16) that

$$
x_{a} \star x_{q}=x_{q_{1}} \cdot x_{j}, \quad \text { for some } q_{1} .
$$

Consider now the sequences of replacements:

$$
\left[x_{a} \cdot x_{j_{0}}\right] \cdot x_{k} \rightarrow\left(x_{q} \cdot x_{j}\right) \cdot x_{k}=x_{q} \cdot\left[x_{j} \cdot x_{k}\right] \rightarrow x_{q} \cdot x_{k^{\prime}} \cdot x_{p} \quad \text { by }(2.20),(2.22)
$$
and

$$
x_{a} \cdot\left[x_{j_{0}} \cdot x_{k}\right] \rightarrow x_{a} \cdot x_{k_{1}} \cdot x_{p},
$$

by the hypothesis of the lemma. It follows from (2.24) and (2.25) that

$$
\left(x_{q} \star x_{k^{\prime}}\right) \star x_{p}=\left(x_{a} \star x_{k_{1}}\right) \star x_{p},
$$

which implies

$$
x_{q} \star x_{k^{\prime}}=x_{a} \star x_{k_{1}},
$$

since $A$ has no zero divisors.

Clearly, the pairs $\left(q, k^{\prime}\right)$ and $\left(a, k_{1}\right)$ are different. By the hypothesis of the lemma, $k_{1}<j_{0}$, hence, since $a>j_{0}$, the equality (2.26) is possible only in case that $q<k^{\prime}$. Thus $(2.26)$ can be written as

$$
x_{a} \star x_{k_{1}}=x_{q} \cdot x_{k^{\prime}}
$$

This, together with (2.16) gives

$$
x_{a} \star x_{q}=x_{q^{\prime}} \cdot x_{k^{\prime}}, \quad \text { for some } q^{\prime} .
$$

Then it follows from (2.23) that

$$
x_{q_{1}} \cdot x_{j}=x_{q^{\prime}} \cdot x_{k^{\prime}} .
$$

Note that this is an equality of two normal monomials. Thus, $j=k^{\prime}$, which contradicts $(2.21)$. We have proved that the inequality $j>j_{0}$ is impossible, hence $j=j_{0}$ and we are done.

(2.27) Proof of the inductive lemma. Assume $j_{0}>1$. Let $k<j_{0}$, and let

$$
x_{j_{0}} \star x_{k}=x_{m} \cdot x_{p}
$$

for some $m$ and $p, p>k, m$. We shall prove that there exists a finite sequence of pairwise different integers

$$
c_{0}=k, c_{1}, \ldots, c_{s-1}, c_{s}=m
$$


such that

$$
\begin{aligned}
& 1 \leq c_{i}<j_{0}, \quad s<j_{0}, \\
& x_{j_{0}} \star x_{c_{i}}=x_{c_{(i-1)}} \cdot x_{p} \quad \text { for } 1 \leq i \leq s \text {, }
\end{aligned}
$$

and

$$
x_{j_{0}} \star x_{k}=x_{c_{s}} \cdot x_{p}
$$

For $i=1$ it follows from Lemma (2.19) (taking the preimage) that there exists a $c_{1}$, uniquely determined by the equalities (2.30) and (2.29). If $c_{1}=$ $k$ we are done. Otherwise, we shall use the following procedure. Assume a sequence of pairwise different integers $c_{1}, \ldots, c_{(r-1)}$ has already been found such that

$$
c_{i} \neq k, \quad \text { for } 1 \leq i<r-1 \text {, }
$$

and (2.29), (2.30) hold for all $i, 1 \leq i \leq r-1$. Furthermore, assume that

$$
c_{(r-1)} \neq k \text {. }
$$

Then, by Lemma (2.19) there exists a $c_{r}$, uniquely determined by the equality (2.30), where $i=r$. Note that

$$
c_{r} \neq c_{i} \text { for all } i, \quad 1 \leq i \leq r-1 .
$$

Indeed, from the equality $c_{r}=c_{i}$ and from (2.30) it follows that

$$
x_{c_{(r-1)}} \cdot x_{p}=x_{j_{0}} \star x_{c_{r}}=x_{j_{0}} \star x_{c_{i}}=x_{c_{(i-1)}} \cdot x_{p}
$$

thus, since the algebra $A$ has no zero divisors, one has $c_{(r-1)}=c_{(i-1)}$, a contradiction with the choice of $c_{1}, \ldots, c_{(r-1)}$. Hence (2.34) holds. If $c_{r}=k$, we are done. Otherwise we continue applying the above procedure. Recall that all the $c_{i}$ 's are pairwise different, and $c_{i}<j_{0}$. It is clear then that in finitely many steps we should find a $c_{s}$, such that $c_{s}=k$. This proves the Inductive Lemma.

(2.35) Proof of Theorem A (1.16). We shall use decreasing induction on $j$. The base of the induction: $n=j$. Similarly to the proof of Lemma 2.23 one can show that if $k<n$ and $x_{n} \star x_{k}=x_{m} \cdot x_{p}$, for some $m$ and $p$ then there is a uniquely determined $c$, such that $x_{n} \star x_{c}=x_{k} \cdot x_{p}$. It is now clear that using the same argument as in the proof of the Inductive Lemma (cf. (2.27)) one can show that the cyclic condition (1.18) holds, but with $j=n$. It is now enough to apply the Inductive Lemma (2.15).

Similarly to the proof of Lemma (2.17) and Corollary (2.18) one can easily see that

(2.36) Remark. Let $k$ be an integer, $k<n$. Then for any $p, k<p \leq n$, there exists a $j, j>k$, such that

$$
x_{j} \star x_{k}=x_{m} \cdot x_{p}, \quad \text { for some } m .
$$

(2.37) Corollary. Let $k, j, p, s=s(k, j)$ be as in (1.18). Then

$$
\left(x_{j}\right)^{s+1} \star x_{k}=x_{k} \cdot\left(x_{p}\right)^{s+1} \text {, }
$$


and therefore, since $s+1$ divides $n !$,

$$
\left(x_{j}\right)^{P} \star x_{k}=x_{k} \cdot\left(x_{p}\right)^{P},
$$

where $P=n !$.

(2.39) Corollary. Suppose that the hypothesis of Theorem A (1.16) is satisfied. Then for $P=n$ ! the following two conditions hold:

(i) For any pair of integers $q, k, 1 \leq k<q \leq n$, there exists $a j, k<$ $j \leq n$, such that

$$
\left(x_{j}\right)^{P} \star x_{k}=x_{k} \cdot\left(x_{q}\right)^{P} \text {. }
$$

(ii) For any pair of integers $j, k, 1 \leq k<j \leq n$, there exists $a p, k<$ $p \leq n$, such that

$$
\left(x_{j}\right)^{P} \star x_{k}=x_{k} \cdot\left(x_{p}\right)^{P} \text {. }
$$

Proof. Assertion (i) follows from Remark (2.36) and Corollary (2.37). The cyclic condition (1.18) and Corollary (2.37) give (ii).

\section{SUfFICIENT CONDITIONS FOR LEFT NOETHERIANNESS}

In this section we shall prove Theorem B of the Introduction. Here the restriction that $A$ has no zero divisors is not necessary. It will be enough to assume as in (1.8), (1.9) that all the coefficients $a_{i j}$ are nonzero. We begin with some preliminary technical results, assuming, as before that the hypotheses of the theorem to be proved are in force, i.e.,

There exists a positive integer $P$, so that the following condition is satisfied:

(3.1) For any pair $q, k, 1 \leq k<q \leq n$ there exists a $j, k<j \leq n$, such that

$$
\left(x_{j}\right)^{P} \star x_{k}=x_{k} \cdot\left(x_{q}\right)^{P} .
$$

It follows immediately from (3.1) that

(3.2) For any pair $j, k, 1 \leq k<j \leq n$ there exists $a q, k<q \leq n$, such that

$$
\left(x_{j}\right)^{P} \star x_{k}=x_{k} \cdot\left(x_{q}\right)^{P} .
$$

(3.3) Definition. (a) Let $u$ and $v$ be two ordered monomials,

$$
u=x_{1}^{s_{1}} \cdots x_{n}^{s_{n}}, \quad s_{i} \geq 0
$$

and

$$
v=x_{1}^{t_{1}} \cdots x_{n}^{t_{n}}, \quad t_{i} \geq 0 .
$$

We say that $v$ is a $P$-multiple of $u$ if for all $i, 1 \leq i \leq n$, one has

$$
t_{i}=s_{i}+r_{i} \cdot P, \quad \text { for some } r_{i} \geq 0 .
$$

(b) We call a normal monomial $w$ a $P$-monomial if $w=x_{1}^{r_{1} P} \cdots x_{n}^{r_{n} P}$, for some $r_{1}, \ldots, r_{n}, r_{i} \geq 0$.

Applying (3.1) and (3.2) one can easily see that 
(3.4) Lemma. If $u$ and $v$ are two normal monomials, and $v$ is a P-multiple of $u$, then there exists a P-monomial $W$ such that $W \star u=v$.

(3.5) Lemma. Let $u$ and $v$ be two normal monomials. Assume that $u<* v$. Let $j$ be an integer, $1 \leq j \leq n$. Then

$$
\left(x_{j}\right)^{P} \star u<*\left(x_{j}\right)^{P} \star v .
$$

Proof. Since the algebra $A$ is graded, it is obvious that (3.6) holds in case that $\operatorname{deg} u<\operatorname{deg} v$. Assume now that $\operatorname{deg} u=\operatorname{deg} v$. It follows from the inequality $u<* v$ that $u=w \cdot x_{i} \cdot f, v=w \cdot x_{k} \cdot g$ for some integers $i, k, 1 \leq i<k \leq n$, and normal monomials $w, f, g$. $(w=1$, or $f=g=1$ is also possible.) Let $x_{j}^{P} \star w=w_{1}$. Two cases arise.

Case 1. The monomial $w_{1} \cdot x_{i}$ is normal. Then, obviously, the monomial $w_{1} \cdot x_{k}$ is normal as well and one has

$$
\left(x_{j}\right)^{P} \star u=w_{1} \cdot x_{i} \cdot f<* w_{1} \cdot x_{k} \cdot g=\left(x_{j}\right)^{P} \star v .
$$

Case 2. The monomial $w_{1} \cdot x_{i}$ is not normal. Applying (3.2), one can easily see in this case that there exists an integer $a, 1<a \leq n$, such that

$$
w_{1}=\left(x_{j}\right)^{P} \star w=w \cdot\left(x_{a}\right)^{P} .
$$

This gives $a>i$. It follows again from (3.2) that

$$
\left(x_{a}\right)^{P} \star x_{i}=x_{i} \star\left(x_{b}\right)^{P}, \quad \text { for some } b>i .
$$

Consider the equalities

$$
\begin{aligned}
\left(x_{j}\right)^{P} \star u & =\left(\left(x_{j}\right)^{P} \star w\right) \star\left(x_{i} \cdot f\right) \\
& =w \cdot\left(\left(\left(x_{a}\right)^{P} \star x_{i}\right) \star f\right) \quad \text { by }(3.7) \\
& =w \cdot x_{i} \cdot\left(\left(x_{b}\right)^{P} \star f\right) \quad \text { by }(3.8) \\
& =w \cdot x_{i} \cdot f_{1}, \quad \text { for } f_{1}=\left(x_{b}\right)^{P} \star f .
\end{aligned}
$$

Hence

$$
\left(x_{j}\right)^{P} \star u=w \cdot x_{i} \cdot f_{1} \in N .
$$

Case 2.a. $k \geq a>i$. Then

$$
\begin{aligned}
\left(x_{j}\right)^{P} \star v & =\left(\left(x_{j}\right)^{P} \star w\right) \star\left(x_{k} \cdot g\right) \\
& =w \cdot\left(\left(\left(x_{a}\right)^{P} \star x_{k}\right) \star g\right) \quad \text { by }(3.7) \\
& =w \cdot\left(x_{a}\right)^{P} \cdot x_{k} \cdot g \\
& =w \cdot x_{a} \cdot g_{1},
\end{aligned}
$$

for $g_{1}=\left(x_{a}\right)^{P-1} \cdot x_{k} \cdot g$. Thus

$$
\left(x_{j}\right)^{P} \star v=w \cdot x_{a} \cdot g_{1} .
$$

Obviously,

$$
w \cdot x_{i} \cdot f_{1}<* w \cdot x_{a} \cdot g_{1} .
$$

This together with (3.9) and (3.10) gives us the desired inequality (3.6).

Case 2.6. $a>k$. It follows from (3.2) that there is a $c, c>k$, such that

$$
\left(x_{a}\right)^{P} \star x_{k}=x_{k} \cdot\left(x_{c}\right)^{P}
$$


and

$$
\left(x_{j}\right)^{P} \star v=w \cdot x_{k} \cdot\left(\left(x_{c}\right)^{P} \star g\right)=w \cdot x_{k} \cdot g_{1},
$$

where $g_{1}=\left(\left(x_{c}\right)^{P} \star g\right)$. If we compare the right-hand sides of the equalities (3.11) and (3.9) we again obtain (3.6).

(3.12) Corollary. Let $u$ and $v$ be normal monomials, such that $u<* v$. Let $W$ be a P-monomial. Then

$$
W \star u<* W \star v .
$$

(3.13) Warning. Note that it is not clear whether for $W, u$, and $v$ as in (3.12) one has $v \star W *>u \star W$.

The following result can easily be obtained from the original Dickson Lemma, cf. [K-R-W].

(3.14) A "P-generalisation" of the Dickson Lemma. Let $u_{1}, u_{2}, \ldots, u_{s}, \ldots$ be a sequence of ordered monomials. Then there exists an integer $i$ such that for any $k>i$, there is $a j=j(k), j \leq i$, such that $u_{k}$ is a P-multiple of $u_{j}$. (3.15) Proof of Theorem B (1.19). Let $J$ be a left ideal in $A$. We shall prove that $J$ is finitely generated as a left ideal. Let $U$ be the set of all highest monomials of the elements of $J$. It is clear that $U$ is a countable set. We can always assume that $U=\left\{u_{1}, u_{2}, \ldots, u_{k}, \ldots\right\}$, where $u_{1}<* u_{2}<* \cdots$ $<* u_{k}<* \cdots$.

By Lemma (3.14), there exists an integer $i_{0}$, such that for any $k>i_{0}$, there is an $i=i(k), i \leq i_{0}$, such that $u_{k}$ is a $P$-multiple of $u_{i}$. Let $f_{1}, f_{2}, \ldots, f_{i_{0}}$ be elements of $J$, with highest monomials respectively $u_{1}, u_{2}, \ldots, u_{i_{0}}$. We can always assume that $f_{i}=u_{i}+g_{i}$, for some polynomial $g_{i}$, such that $H M\left(g_{i}\right)$ $<* u_{i}$. We shall prove that the polynomials $f_{1}, \ldots, f_{i_{0}}$ generate $J$ as a left ideal. Let $J_{0}$ be the left ideal, generated by $f_{1}, \ldots, f_{i_{0}}$. Obviously $J_{0} \subseteq J$. Assume that $J_{0} \neq J$. Let $f \in J \backslash J_{0}$ be a polynomial with minimal highest monomial. By the definition of $U$ there is a $k$, such that $u_{k}$ is the highest monomial of $f$, i.e., $f=c \cdot u_{k}+g$, where $c \in K, g=a_{1} \cdot v_{1}+\cdots+a_{s} \cdot v_{s}$, and $v_{j}<* u_{k}$, for $1 \leq j \leq s$. We can always assume that $c=1$. By the choice of $i_{0}$, there exists an $i, i \leq i_{0}$, such that $u_{k}$ is a $P$-multiple of $u_{i}$.

It follows from Lemma (3.4) that there exists a normal $P$-monomial $W$ such that $W \star u_{i}=u_{k}$. Note that by Corollary (3.12), $W \star u_{i}$ is the highest monomial of the polynomial $\operatorname{Nor}\left(W \cdot f_{i}\right)$, where $\operatorname{Nor}\left(W \cdot f_{i}\right)$ is the normal form of the element $W \cdot f_{i}$. Consider now the polynomial

$$
h=f-\frac{1}{\alpha} \operatorname{Nor}\left(W \cdot f_{i}\right),
$$

where $\alpha$ is the coefficient of the highest monomial of $\operatorname{Nor}\left(W \cdot f_{i}\right)$. It is clear that $h \in J \backslash J_{0}$, and $h$ is a nonzero polynomial with highest monomial strictly less than $u_{k}$. This contradicts the choice of $f$. We have proved that $J_{0}=J$. Hence, $A$ is left Noetherian.

(3.16) Remark. If $A$ has no zero divisors then conditions (1.17) and (1.20) from the introduction are equivalent.

Proof. Clearly (1.20) implies (1.17). The implication (1.17) $\Rightarrow(1.20)$ follows from Corollary (2.39).

Note that, if $A$ has no zero divisors and satisfies (1.20), then $A$ is cyclic. (This follows from Remark (3.16) and Theorem A (1.16).) 
(3.17) Corollary. Let $A=K\left\langle x_{1}, \ldots, x_{n}\right\rangle /(F)$ be a binomial skew polynomial ring without zero divisors, with reduced Groebner basis

$$
F=\left\{x_{j} x_{i}-a_{i j} x_{i^{\prime}} x_{j^{\prime}} \mid 1 \leq i<j \leq n\right\},
$$

where for $1 \leq i<j \leq n$ one has $1 \leq i^{\prime}<j^{\prime} \leq n$, and $x_{i^{\prime}} x_{j^{\prime}}<* x_{j} x_{i}$. Suppose furthermore that

$$
\text { The set }\left\{x_{i^{\prime}} x_{j^{\prime}} \mid 1 \leq i<j \leq n\right\} \text { contains precisely }\left(\begin{array}{l}
n \\
2
\end{array}\right) \text { elements. }
$$

Then $A$ is left Noetherian.

Proof. It follows from Corollary (2.39) and Remark (3.16) that for $P=n !, A$ satisfies the hypotheses of Theorem B. Hence $A$ is left Noetherian.

\section{LEFT AND RIGHT NOETHERIANNESS}

In this section we shall prove Theorem C (1.21) of the Introduction. We assume that the hypotheses of the theorem to be proved are in force, i.e.,

(4.1) $A=K\left\langle x_{1}, \ldots, x_{n}\right\rangle /(F)$ is a binomial skew polynomial ring without zero divisors, with reduced Groebner basis

$$
F=\left\{x_{j} x_{i}-a_{i j} x_{i^{\prime}} x_{j^{\prime}} \mid 1 \leq i<j \leq n\right\},
$$

where for $1 \leq i<j \leq n$ one has $1 \leq i^{\prime}<j^{\prime} \leq n$, and $x_{i^{\prime}} x_{j^{\prime}}<* x_{j} x_{i}$.

We begin with some technical results.

The following lemma is true even if the condition (1.10) is not satisfied.

(4.2) Lemma. Let A be a binomial skew polynomial ring without zero divisors. Then the following holds:

(a) If $A$ is left Noetherian then for any $i, j, 1 \leq i<j \leq n$, there exists a normal monomial $w$ such that

$$
w \star x_{i}=x_{i} \cdot x_{j}^{\operatorname{deg} w} .
$$

(b) If $A$ is right Noetherian, then for any $i, j, 1 \leq i<j \leq n$, there exists a normal monomial $w$ such that

$$
x_{j} \star w=x_{i}^{\operatorname{deg} w} \cdot x_{j} .
$$

Proof. (a) Assume $A$ is left Noetherian and $j>i$. Consider the increasing chain of left ideals $I_{1} \subseteq I_{2} \subseteq \cdots \subseteq I_{k} \subseteq \cdots$, where for $k \geq 1, I_{k}$ is the left ideal generated by the elements $x_{i} x_{j}, x_{i} x_{j}^{2}, \ldots, x_{i} x_{j}^{k}$. It follows from the left Noetherianness of $A$ that there exists a $k$ such that $I_{k}=I_{k+1}=\cdots$. This implies that $x_{i} x_{j}^{k+1} \in I_{k}$. Hence the following equality holds:

$$
x_{i} x_{j}^{k+1}-\sum_{q} \sum_{1 \leq r \leq k} b_{r q} w_{r q} \star x_{i} x_{j}^{r}=0,
$$

where the $w_{r q}$ are normal monomials and the $b_{r q}$ are nonzero elements of $K$. This equality gives that a linear combination of nonzero normal monomials is zero. This is possible only in case all the coefficients in the equality (4.3) are zero, which implies that $x_{i} x_{j}^{k+1}=w_{r q} \star x_{i} x_{j}^{r}$ for some $q$ and $r, r \leq k$. Since 
$A$ has no zero divisors this implies that $x_{i} x_{j}^{k+1-r}=w_{r q} \star x_{i}$. We have proved (4.2(a). A similar argument shows that (4.2(b)) also holds.

(4.4) Lemma. Let $f$ be an arbitrary monomial which is not normal. Assume the normal form of $f$ is either (a) $w=x_{i} \cdot\left(x_{j}\right)^{t}$, or (b) $w=\left(x_{i}\right)^{t} \cdot x_{j}$, where in both cases $1 \leq i<j \leq n$ and $t>1$. Then there exists a pair of iniegers $p, q$, $1 \leq p<q \leq n$, such that $x_{q} \star x_{p}=x_{i} \cdot x_{j}$.

Proof. This follows from condition (1.10).

(4.5) Corollary. Let $A=K\left\langle x_{1}, \ldots, x_{n}\right\rangle /(F)$ be a binomial skew polynomial ring, with reduced Groebner basis

$$
F=\left\{x_{j} x_{i}-a_{i j} x_{i^{\prime}} x_{j^{\prime}} \mid 1 \leq i<j \leq n\right\},
$$

where for $1 \leq i<j \leq n$ one has $1 \leq i^{\prime}<j^{\prime} \leq n$, and $x_{i^{\prime}} x_{j^{\prime}}<* x_{j} x_{i}$. Suppose furthermore that $A$ is left (or right) Noetherian. Then the following two equivalent conditions hold:

(a) The set $\left\{x_{i^{\prime}} x_{j^{\prime}} \mid 1 \leq i<j \leq n\right\}$ contains precisely $\left(\begin{array}{c}n \\ 2\end{array}\right)$ elements.

(b) For any pair of integers $i, j, 1 \leq i<j \leq n$, there exist uniquely determined $q$ and $p, 1 \leq p<q \leq n$, such that $x_{q} \star x_{p}=x_{i} \cdot x_{j}$.

Proof. Case 1. $A$ is left Noetherian. Consider the sets

$$
\begin{aligned}
& P_{1}=\left\{x_{j} \star x_{i} \mid 1 \leq i<j \leq n\right\}=\left\{x_{i}^{\prime} \cdot x_{j}^{\prime} \mid 1 \leq i<j \leq n\right\}, \\
& P_{2}=\left\{x_{i} \cdot x_{j} \mid 1 \leq i<j \leq n\right\} .
\end{aligned}
$$

Obviously

$$
P_{1} \subseteq P_{2}
$$

We shall show that there is an equality of sets in (4.6). Indeed, take an arbitrary pair $i, j, 1 \leq i<j \leq n$. It follows from Lemma (4.2a) that there exists a normal monomial $w$ such that

$$
w \star x_{i}=x_{i} \cdot\left(x_{j}\right)^{\operatorname{deg} w} .
$$

It then follows from Lemma (4.4) that $x_{i} \cdot x_{j}=x_{q} \star x_{p}$, for some $p$ and $q$, $1 \leq p<q \leq n$. Thus $P_{2} \subseteq P_{1}$, which together with (4.6) gives $P_{1}=P_{2}$ and

$$
\operatorname{Card}\left(P_{1}\right)=\operatorname{Card}\left(P_{2}\right)=\left(\begin{array}{l}
n \\
2
\end{array}\right) .
$$

This proves $(4.5(\mathrm{a}))$ and $(4.5(\mathrm{~b}))$.

Case 2. $A$ is right Noetherian. Apply Lemmas (4.2(b)), (4.4(b)).

Recall that given an algebra $A$ with product written as $u \cdot v, A^{\text {op }}$ is the algebra based on the same underlying vector space, with product written as $u \circ v=v \cdot u$. Clearly, $A$ is left Noetherian if and only if $A^{\text {op }}$ is right Noetherian. Also, if $A=K\langle X\rangle /(F)$, where $(F)$ is as in (4.1), is a binomial skew polynomial ring, then $A^{\text {op }}=K\langle x\rangle /\left(F^{\text {op }}\right)$, where

$$
F^{\mathrm{op}}=\left\{x_{j^{\prime}} x_{i^{\prime}}-a_{i j}^{-1} x_{i} x_{j} \mid 1 \leq i<j \leq n\right\} .
$$

In general, the fact that $F$ is a Groebner basis does not imply that $F^{\text {op }}$ is a Groebner basis, as one can check in example (1.12). 
(4.8) Proposition. Let $A=K\left\langle x_{1}, \ldots, x_{n}\right\rangle /(F)$ be a binomial skew polynomial ring, with reduced Groebner basis

$$
F=\left\{x_{j} x_{i}-a_{i j} x_{i^{\prime}} x_{j^{\prime}} \mid 1 \leq i<j \leq n\right\}
$$

where for $1 \leq i<j \leq n$ one has $1 \leq i^{\prime}<j^{\prime} \leq n$, and $x_{i^{\prime}} x_{j^{\prime}}<* x_{j} x_{i}$. Suppose furthermore that

$$
\text { The set }\left\{x_{i^{\prime}} x_{j^{\prime}} \mid 1 \leq i<j \leq n\right\} \text { contains precisely }\left(\begin{array}{l}
n \\
2
\end{array}\right) \text { elements. }
$$

Then the following three conditions hold:

(a) $F^{\text {op }}$ is the reduced Groebner basis of the ideal $\left(F^{\mathrm{op}}\right)$.

(b) $A^{\text {op }}$ is a binomial skew polynomial ring.

(c) $A^{\text {op }}$ is left Noetherian.

Proof. It follows from (4.7) that all the elements of $F^{\text {op }}$ are binomials of the type

$$
g_{i^{\prime} j^{\prime}}=x_{j^{\prime}} x_{i^{\prime}}-a_{i j}^{-1} x_{i} x_{j}
$$

where $1 \leq i<j \leq n, 1 \leq i^{\prime}<j^{\prime} \leq n$. Lemma (2.3) shows that for any pair $i^{\prime}, j^{\prime}, 1 \leq k^{\prime}<j^{\prime} \leq n$, one has $i<j^{\prime}$, hence

$$
x_{j^{\prime}} x_{i^{\prime}}=H M\left(g_{i^{\prime} j^{\prime}}\right),
$$

where $H M\left(g_{i^{\prime} j^{\prime}}\right)$ is the highest monomial of $g_{i^{\prime} j^{\prime}}$, cf. (1.1). Recall now (cf. [G-I $\left.I_{1}\right]$ ) that given an ideal $I$ in $K\langle X\rangle$ a monomial $u$ is normal modulo $I$ if it does not contain as a subword any of the monomials $H M(f)$, where $f \in I$. Let $N(I)$ be the set of all normal modulo $I$ monomials. It is known, cf. [Berg, G-I ${ }_{1}$, that $N(I)$ projects to a $K$-basis of the algebra $B=K\langle X\rangle / I$. Now for $I=\left(F^{\mathrm{op}}\right)$ it is clear that

$$
N(I) \subseteq N
$$

where as in (1.1), $N$ is the set of ordered monomials. We shall prove that equality holds in (4.11). Recall that

(4.12) The algebras $A$ and $A^{\text {op }}$ have the same Hilbert series.

(4.13) $N$ projects to a $K$-basis of $A=K\langle X\rangle /(F), N(I)$ projects to a $K$-basis of $A^{\text {op }}=K\langle X\rangle /(I)$.

It follows from (4.10), (4.12), and (4.13) that

$$
N\left(F^{\text {op }}\right)=N(I)=N \text {. }
$$

It then follows from the Diamond Lemma [Berg] (cf. also (1.6)) that $F^{\text {op }}$ is a Groebner basis of the ideal $\left(F^{\mathrm{op}}\right)$. This proves $(4.8 \mathrm{a})$. Obviously (4.8a) implies (4.8b).

Consider now the set

$$
F^{\text {op }}=\left\{x_{j^{\prime}} x_{i^{\prime}}-a_{i j}^{-1} x_{i} x_{j} \mid 1 \leq i<j \leq n, 1 \leq i^{\prime}<j^{\prime} \leq n\right\} .
$$

It follows from (4.10) that there is an equality

$$
x_{\left(i^{\prime}\right)^{\prime}} x_{\left(j^{\prime}\right)^{\prime}}=x_{i} x_{j}
$$

for all $i^{\prime}, j^{\prime}, 1 \leq i^{\prime}<j^{\prime} \leq n$. Obviously, the set

$$
\left\{x_{\left(i^{\prime}\right)^{\prime}} x_{\left(j^{\prime}\right)^{\prime}} \mid 1 \leq i^{\prime}<j^{\prime} \leq n\right\}=\left\{x_{i} x_{j} \mid 1 \leq i<j \leq n\right\}
$$


contains precisely $\left(\begin{array}{l}n \\ 2\end{array}\right)$ elements. It follows from here that $A^{\text {op }}$ satisfies the hypotheses of Corollary (3.17), hence, it is left Noetherian.

(4.14) Proof of Theorem C (1.21).

Corollary (3.17) gives the implication (i) $\Rightarrow$ (ii) .

(i) $\Rightarrow$ (iii). It follows from Proposition $(4.8(\mathrm{c}))$ that $A^{\text {op }}$ is left Noetherian, hence $A$ is right Noetherian.

Corollary $(4.5(\mathrm{a}))$ shows that $(\mathrm{ii}) \Rightarrow$ (i) and (iii) $\Rightarrow$ (i) .

(4.15) Definition. For the set of semigroup relations

$$
G=\left\{x_{j} \cdot x_{i}=x_{i^{\prime}} x_{j^{\prime}} \mid 1 \leq i<j \leq n\right\}
$$

where for $1 \leq i<j \leq n$ one has $1 \leq i^{\prime}<j^{\prime} \leq n$, and $i^{\prime}<j$, we say that $G$ is a semigroup Groebner basis if and only if the corresponding set of relations

$$
F(G)=F=\left\{x_{j} \cdot x_{i}-x_{i^{\prime}} x_{j^{\prime}} \mid 1 \leq i<j \leq n\right\}
$$

is a Groebner basis of the ideal $(F)$ in $K\langle X\rangle$.

(4.16) Corollary. Let $S=\langle X \mid G\rangle$ be a semigroup with set of generators $X$ and set of relations

$$
G=\left\{x_{j} x_{i}=x_{i^{\prime}} x_{j^{\prime}} \mid 1 \leq i<j \leq n\right\},
$$

where for $1 \leq i<j \leq n$ one has $1 \leq i^{\prime}<j^{\prime} \leq n$, $i^{\prime}<j$. Suppose $G$ is a semigroup Groebner basis. The the following three conditions are equivalent:

(i) The set $\left\{x_{i^{\prime}} x_{j^{\prime}} \mid 1 \leq i<j \leq n\right\}$ contains precisely $\left(\begin{array}{l}n \\ 2\end{array}\right)$ elements.

(ii) $S$ is left Noetherian.

(iii) $S$ is right Noetherian.

We shall finish with a full list of left and right Noetherian binomial skew polynomial rings with three and four generators. It turns out that

(4.17) In this case the condition (1.10), $i^{\prime}<j^{\prime}$ for any $i<j$ appears as a consequence of left Noetherianness.

By $F_{1}$ we shall denote a subset of $K\langle X\rangle$ of the type

$$
F_{1}=\left\{x_{j} x_{i}-a_{j i} x_{i^{\prime}} x_{j^{\prime}} \mid 1 \leq i<j \leq n, 1 \leq i^{\prime} \leq j^{\prime} \leq n\right\} \text {. }
$$

Note that in difference with $F$, cf. (1.9), (1.10), we do not assume $i^{\prime} \neq j^{\prime}$. One can easily check using the Groebner basis property $(1.4(\mathrm{~b}))$ that

(4.19) Lemma. Let $A=K\left\langle x_{1}, x_{2}, x_{3}\right\rangle /\left(F_{1}\right)$ be a binomial skew polynomial ring. Then $A$ is left (respectively right) Noetherian if and only if one of the following conditions hold:

(1) $F_{1}=\left\{x_{3} x_{2}-a x_{2} x_{3}, x_{3} x_{1}-b x_{1} x_{3}, x_{2} x_{1}-c x_{1} x_{2} \mid a b c \neq 0\right\}$.

(2) $F_{1}=\left\{x_{3} x_{2}-a x_{1} x_{3}, x_{3} x_{1}-b x_{2} x_{3}, x_{2} x_{1}-c x_{1} x_{2} \mid c^{2}=1, a b \neq 0\right\}$.

(3) $F_{1}=\left\{x_{3} x_{2}-a x_{2} x_{3}, x_{3} x_{1}-b x_{1} x_{2}, x_{2} x_{1}-c x_{1} x_{3} \mid a^{2}=1, b c \neq 0\right\}$.

Some more delicate combinatorial arguments show that the following proposition holds. 
(4.20) Proposition. Let $A=K\left\langle x_{1}, x_{2}, x_{3}, x_{4}\right\rangle /\left(F_{1}\right)$ be a binomial skew polynomial ring. Then $A$ is left (respectively right) Noetherian if and only if one of the following conditions holds:

$$
\begin{aligned}
& F_{1}=\left\{x_{4} x_{3}-a x_{1} x_{4}, x_{4} x_{2}-b x_{3} x_{4}, x_{4} x_{1}-c x_{2} x_{4}, x_{3} x_{2}-d x_{2} x_{3},\right. \\
& \left.x_{3} x_{1}-d^{-1} x_{1} x_{3}, x_{2} x_{1}-d x_{1} x_{2} \mid a b c d \neq 0\right\}, \\
& F_{1}=\left\{x_{4} x_{3}-a x_{2} x_{4}, x_{4} x_{2}-b x_{1} x_{4}, 4 x_{4} x_{1}-c x_{3} x_{4},\right. \\
& F_{1}=\left\{x_{4} x_{3}-a x_{2} x_{4}, x_{4} x_{2}-b x_{3} x_{4}, x_{4} x_{1}-c x_{1} x_{4}, x_{3} x_{2}-d x_{2} x_{3},\right. \\
& x_{3} x_{1}-e x_{1} x_{2}, x_{2} x_{1}-f x_{1} x_{3} \mid d^{2}=1 \\
& a f=b e, \text { abcdef } \neq 0\},
\end{aligned}
$$

$F_{1}=\left\{x_{4} x_{3}-a x_{2} x_{4}, x_{4} x_{2}-b x_{3} x_{4}, x_{4} x_{1}-c x_{1} x_{4}, x_{3} x_{2}-d x_{2} x_{3}\right.$ $x_{3} x_{1}-e x_{1} x_{3}, x_{2} x_{1}-e x_{1} x_{2} \mid d^{2}=1$, abcde $\left.\neq 0\right\}$,

$$
\begin{aligned}
& F_{1}=\left\{x_{4} x_{3}-a x_{1} x_{4}, x_{4} x_{2}-b x_{2} x_{4},\right. \\
& x_{4} x_{1}-c x_{3} x_{4}, x_{3} x_{2}-d x_{2} x_{3} \text {, } \\
& \left.x_{3} x_{1}-e x_{1} x_{3}, x_{2} x_{1}-d^{-1} x_{1} x_{2} \mid e^{2}=1, a b c d \neq 0\right\} \text {, } \\
& F_{1}=\left\{x_{4} x_{3}-a x_{3} x_{4}, x_{4} x_{2}-b x_{1} x_{3}, x_{4} x_{1}-c x_{2} x_{3}, x_{3} x_{2}-d x_{1} x_{4},\right. \\
& x_{3} x_{1}-e x_{2} x_{4}, x_{2} x_{1}-f x_{1} x_{2} \mid a b c d e f \neq 0, \\
& \left.a^{2}=f^{2}=b e / c d=c d / b e\right\},
\end{aligned}
$$$$
F_{1}=\left\{x_{4} x_{3}-a x_{3} x_{4}, x_{4} x_{2}-b x_{2} x_{3}, x_{4} x_{1}-c x_{1} x_{3}, x_{3} x_{2}-d x_{2} x_{4}\right. \text {, }
$$$$
x_{3} x_{1}-e x_{1} x_{4}, x_{2} x_{1}-f x_{1} x_{2} \mid a^{2}=1 \text {, }
$$$$
b e=c d, c d f \neq 0\},
$$

$$
\begin{array}{r}
F_{1}=\left\{x_{4} x_{3}-a x_{3} x_{4}, x_{4} x_{2}-b x_{1} x_{4}, x_{4} x_{1}-c x_{2} x_{4}, x_{3} x_{2}-d x_{1} x_{3},\right. \\
x_{3} x_{1}-e x_{2} x_{3}, x_{2} x_{1}-f x_{1} x_{2} \mid f^{2}=1, \\
b e=c d, a c d \neq 0\},
\end{array}
$$

$$
\begin{aligned}
& F_{1}=\left\{x_{4} x_{3}-a x_{3} x_{4}, x_{4} x_{2}-b x_{1} x_{4}, x_{4} x_{1}-c x_{2} x_{4}, x_{3} x_{2}-d x_{2} x_{3}\right. \text {, } \\
& \left.x_{3} x_{1}-d x_{1} x_{3}, x_{2} x_{1}-f x_{1} x_{2} \mid f^{2}=1, a b c d \neq 0\right\}, \\
& F_{1}=\left\{x_{4} x_{3}-a x_{3} x_{4}, x_{4} x_{2}-b x_{2} x_{4}, x_{4} x_{1}-c x_{1} x_{2}, x_{3} x_{2}-a x_{2} x_{3},\right. \\
& \left.x_{3} x_{1}-e x_{1} x_{4}, x_{2} x_{1}-f x_{1} x_{3} \mid a b=1 \text {, cef } \neq 0\right\}, \\
& F_{1}=\left\{x_{4} x_{3}-a x_{3} x_{4}, x_{4} x_{2}-b x_{2} x_{4}, x_{4} x_{1}-c x_{1} x_{3}, x_{3} x_{2}-a x_{2} x_{3}\right. \text {, } \\
& \left.x_{3} x_{1}-e x_{1} x_{2}, x_{2} x_{1}-f x_{1} x_{4} \mid a b=1 \text {, cef } \neq 0\right\},
\end{aligned}
$$




$$
\begin{aligned}
& F_{1}=\left\{x_{4} x_{3}-a x_{3} x_{4}, x_{4} x_{2}-b x_{2} x_{4}, x_{4} x_{1}-c x_{1} x_{2},\right. \\
& x_{3} x_{2}-d x_{2} x_{3}, x_{3} x_{1}-e x_{1} x_{3} \text {, } \\
& \left.x_{2} x_{1}-f x_{1} x_{4} \mid a d=b^{2}=1 \text {, cef } \neq 0\right\} \text {, } \\
& F_{1}=\left\{x_{4} x_{3}-a x_{3} x_{4}, x_{4} x_{2}-b x_{2} x_{4}, x_{4} x_{1}-c x_{1} x_{3}, x_{3} x_{2}-b x_{2} x_{3}\right. \text {, } \\
& \left.x_{3} x_{1}-e x_{1} x_{4}, x_{2} x_{1}-f x_{1} x_{2} \mid a^{2}=1, \text { bce } \neq \neq 0\right\} \text {, } \\
& F_{1}=\left\{x_{4} x_{3}-a x_{3} x_{4}, x_{4} x_{2}-b x_{2} x_{4}, x_{4} x_{1}-c x_{1} x_{4}, x_{3} x_{2}-d x_{2} x_{3}\right. \text {, } \\
& \left.x_{3} x_{1}-e x_{1} x_{3}, x_{2} x_{1}-f x_{1} x_{2} \mid \text { abcde } f \neq 0\right\} \text {, } \\
& F_{1}=\left\{x_{4} x_{3}-a x_{3} x_{4}, x_{4} x_{2}-b x_{2} x_{4}, x_{4} x_{1}-b x_{1} x_{4}, x_{3} x_{2}-d x_{1} x_{3}\right. \text {, } \\
& \left.x_{3} x_{1}-e x_{2} x_{3}, x_{2} x_{1}-f x_{1} x_{2} \mid f^{2}=1, \text { abde } \neq 0\right\} \text {, } \\
& F_{1}=\left\{x_{4} x_{3}-a x_{3} x_{4}, x_{4} x_{2}-a x_{2} x_{4}, x_{4} x_{1}-c x_{1} x_{4}, x_{3} x_{2}-d x_{2} x_{3}\right. \text {, } \\
& \left.x_{3} x_{1}-e x_{1} x_{2}, x_{2} x_{1}-f x_{1} x_{3} \mid d^{2}=1, \text { acf } \neq 0\right\}, \\
& F_{1}=\left\{x_{4} x_{3}-a x_{3} x_{4}, x_{4} x_{2}-b x_{2} x_{3}, x_{4} x_{1}-c x_{1} x_{4},\right. \\
& x_{3} x_{2}-d x_{2} x_{4}, x_{3} x_{1}-c x_{1} x_{3} \text {, } \\
& \left.x_{2} x_{1}-f x_{1} x_{2} \mid a^{2}=1, b c d f \neq 0\right\} \text {. }
\end{aligned}
$$

\section{BIBLIOGRAPHY}

[Ap] J. Apel, Gröbnerbasen in nichtkommutativen Algebren und ihre anwendung, Dissertation A, Karl-Marx-Universität, Leipzig.

[Art-S] M. Artin and W. Schelter, Graded algebras of global dimension 3, Adv. Math. 66 (1987), 171-216.

[Berg] G. M. Bergman, The diamond lemma of ring theory, Adv. Math. 29 (1978), 178-218.

[Buch] B. Buchberger, An algorithm for finding a basis for the residue class ring of a zero-dimensional polynomial ideal, Ph.D. thesis, Univ. Innsbruck (Austria), Math. Inst., 1965, Aequationes Math. 4 (1970), 374-383. (German)

[G-I $\mathrm{I}_{1}$ ] T. Gateva-Ivanova, On the Noetherianity of some associative finitely presented algebras, J. Algebra 138 (1991), 13-35.

[G-I $\mathrm{I}_{2}$ ] _ Noetherian properties and growth of some associative algebras, Progr. Math., vol. 94, Birkhäuser, Basel and Boston, 1991, pp. 143-158.

[G-I $\left.{ }_{3}\right]$ _ Groebner bases in skew polynomial rings, preprint, Dept. of Math. Reports No. 8, University of Stockholm, 1992, pp. 1-33.

[Go1] E. S. Golod, Standard bases and homology, Lecture Notes in Math., vol. 1352, SpringerVerlag, Berlin and New York, 1988, pp. 88-95.

[K-R-W] A. Kandri-Rody and V. Weispfenning, Non-commutative Gröbner bases in algebras of solvable type, J. Symbolic Comput. 9 (1990), 1-26.

[Mor ${ }_{1}$ ] T. Mora, Groebner bases for non-commutative algebras, ISSAC 88, Lecture Notes in Computer Science, vol. 358, Springer-Verlag, Berlin and New York, 1989, pp. 150-161.

[Mor ${ }_{2}$ ] T. Mora, Seven variations on standard bases, Univ. di Genova, Dipartimento di Matematica, N. 45 Preprint, 1988.

[Sm-St] S. P. Smith and J. T. Stafford, Regularity of the four dimensional Sklyanin algebra, preprint, 1990.

Institute of Mathematics, Bulgarian Academy of Sciences, Sofia 1113, Bulgaria

E-mail address: tatiana@bgearn. bitnet 